\title{
Review
}

Gene M. Remmers

Ship Strutures and Systems Science and Technology Division Office of Naval Research Arlington, VA 22217-5660

George J. O'Hara

Department of Mechanical Engineering

University of Maryland College Park, MD 20742

Patrick F. Cunniff

Department of Mechanical Engineering

University of Maryland College Park, MD 20742

\section{Dynamic Design Analysis Method DDAM}

This article describes the evolution of the dynamic design analysis method (DDAM) by assimilating information from references spanning more than three decades. This evolution began with attempts to use earthquake engineering practice, circa 1950, in dealing with hostile environments created by modern weaponry. It became necessary to develop new theories that went beyond the then current status. This led to research programs that went back to basic physics and engineering principles that resulted in a sound technique for naval applications. The elements of the technique were theoretically based and confirmed by laboratory and large scale field testing. One important example is the structural interaction effects between a vehicle and large equipment structures by means of a newly defined quantity called modal effective mass. Another example led to the discovery that attaching a vibration generator to a structure in an effort to find the frequencies useful for foundation motion response analysis was guaranteed to produce failure. DDAM continues to be used after its introduction 36 years ago. Although familiar in US and international naval circles, it is not well known by persons other than naval engineers. Many myths and misconceptions have grown during this period, so some of the major ones are addressed. (C) 1996 John Wiley \& Sons, Inc.

\section{INTRODUCTION}

Even though the dynamic design analysis method (DDAM) has been in widespread use in the naval and marine engineering community for many years, newcomers and engineers from other disciplines are sometimes unfamiliar with its physical basis. It differs from other (shock) spectral analysis methods in several respects: the equipment structure itself is used to account for equipmentvehicle interaction effects and to determine the equipment shock design input values; it uses structural information to determine the number of modes needed in the analysis; it permits analysis of up to 6 degrees of freedom for each mass point, even though the shocks are unidirectional; it has a special summation procedure to evaluate critical values; and it has a mechanism to permit small amounts of plastic yielding in those cases where it is safe to do so. This article presents the highlights of the technique, particularly in those areas where differences with other methods oc-

Received May 7, 1996; Accepted June 20, 1996.

Shock and Vibration, Vol. 3, No. 6, pp. 461-476 (1996)

(C) 1996 by John Wiley \& Sons, Inc.

CCC 1070-9622/96/060461-16 
cur. Emphasis was and is placed on the engineering background research to ensure close adherence to reality and the principles of physics and to ensure that experimental validation concurred. Testing and experience also confirmed and validated the method.

Consider the situation created by a nuclear or chemical underwater explosion attack upon a surface ship or submarine. There is within the vessel myriad equipment and machinery that must continue to function if the vessel is to survive and fight. The experiences of World War II, including the noncontact influence mine and the development of nuclear weapons, made it imperative to shock harden both ships and submarines. In the early 1950 s there were only a few shock test machines that were available to provide some degree of shock hardness for lightweight equipment weighing less than 3-4 tons. Even then, some of these lightweight items had a physical size and geometric configuration that were beyond any practical capability to design by test. The use of nuclear power also contributed to the needs of expanding the testing facilities and design analysis techniques. Combining the techniques of that era, as used by some aircraft designers and manufacturers (design, test, modify design, test again, etc.), seemed to be prohibitively expensive when applied to large naval structures because the range of attacks that define the operational environment to be survived are extremely harsh and varied. The need to analyze equipment became a very important part of the effort.

A group of engineers and scientists at the Naval Research Laboratory (NRL) was directed to address this problem and to propose analytical procedures to shock harden naval equipment. The then current procedures (circa early 1950s) of the earthquake engineers was first investigated. As pointed out later in this article, it was found to be wanting in several respects. A research program was conducted and DDAM was the result (Belsheim and O'Hara, 1960, 1961; O'Hara and Belsheim, 1961, 1963).

\section{DESIGN ANALYSIS}

Because critical machinery and equipment have a function to perform, the engineer/analyst has a special problem. Turbines, bull gears, and shafts are some examples where function drives the design; yet they must be hardened to survive battle conditions, which is a formidable task. What then is a DDAM? It must contain several salient parts:

1. It must recognize that equipment function controls much of the configuration.

2. Failure criteria involving such things as collision clearances and alignment sensitivity may be as important as stress and deflection. These must be capable of being calculated by the design method.

3. A mathematical description of the equipment structure must be possible to permit comparisons with failure criteria. DDAM uses normal mode theory.

4. A description of the shock loading environment needs to be provided that is attuned to the equipment and that is neither academic nor extremely costly to use. The description should include the ability to modify the shock design values in order to distinguish between structures and equipment of widely varied mass and mobility characteristics. Design must be possible, even for conditions that make many other shock events, such as earthquakes, seem very mild.

\section{EVOLUTION OF DDAM}

For many years engineers have used "shock response spectra" as an aid in understanding the damaging potential of shock, and as a tool for stress checking a structure whose foundation was subjected to the transient for which the spectrum was found.

\section{Shock Response Spectrum Definition}

Because shock response spectrum is a term that various authors have used in different ways, the explicit definition used here is provided. It is the plot of the maximum absolute values of the relative displacements, multiplied by scaling factors if desired, of a set of either damped or undamped single degree of freedom oscillators with negligible mass that have been subjected to a shock motion, the values being plotted as a function of the natural frequencies of the simple oscillators. These graphs can be constructed with units of displacement, velocity, or equivalent static acceleration by choice of the scaling factors, unity, $\omega$, or $\omega^{2} / g$, respectively, where $\omega$ is the angular frequency of the oscillator. 


\section{Initial Development}

One thing that became apparent very early was that even though each earthquake or shock is unique, the way a structure fails is not. Among the approaches followed was that pioneered by many earthquake structural analysts including M. A. Biot. An account of this early portion of earthquake structural engineering and the Navy's attempt to use it are described in the Biot Memorial Lecture by Remmers (1983). Great difficulties were encountered with attempting to apply the earthquake methods to naval design problems in the area of describing the environment via envelopes of response spectra, typical shock motions, averaged motions, and averaged spectra. As described below, these were found to not be useful.

In a set of field trials, data were collected for a number of shocks at a large number of different points in ships and submarines. It was hoped that by classifying the location, intensity of shock, and equipment responding to those shocks, correlated groups could be formed that would enable the analyst to produce a series of shock spectra that would be useful in design. Envelope type shock spectra were produced, and it immediately became apparent that few structures would survive the severe shock these spectra would demand. However, the structures that were in place during field trials did survive, so it was deduced that something must have been wrong with the theory of combining these shock spectra.

Inherent in the assumption that the worst possible shock that a class of structures will ever have to undergo is the envelope of all possible shock spectra for that type of location is the following: impedance of the vehicle at the base of the equipment attachment point is very large over the entire frequency range, and the equipment has very little impedance at its base over the frequency range. This means that the dynamic reaction of the equipment upon the base (attachment point to the vehicle) is unable to affect the motion of the base, and dissimilar equipment structures will have to respond to the same base motion. Of course this assumption is obviously not in accordance with physical reality. (A discussion of mechanical impedance and mobility can be found in O'Hara, 1959b, 1967.)

During the mid 1950s, examination of individual shock spectra showed large valleys in the region of these fixed-based frequencies (Belsheim and Blake, 1957). This was labeled the shock spectrum dip effect. It was the subject of intensive effort during this era and was confirmed theoretically by O'Hara (1959a). The problem of overconservatism of such envelope and/or fiducial limit curves was investigated, and it was then noticed that computing stress with normal mode theory only requires shock spectrum values at the fixed-base natural frequencies of the equipment in place during the shock motion. A summary of this and other work on this subject can be found in O'Hara and Cunniff (1985).

The first experiment designed to verify this spectrum dip effect was performed at NRL on the medium weight shock machine and reported by O'Hara $(1958,1961)$. This was later reconfirmed in a new and different experiment by O'Hara and Sweet (1960).

The original experiment was performed to study the effect of changes in equipment structural parameters such as mass and stiffness upon shock spectra. Hence, an experimental equipment structure (Fig. 1) was designed and built such that it was possible to markedly change the stiffness of this equipment without changing its mass, and also to change the mass while retaining the same stiffness, so the effects of varying both parameters could be measured. It was designed to have two different stiffness configurations with a 6-mass incremental variation in each. The same hammer blow to a Navy medium weight shock machine anvil was used in each test to maintain the same shock intensity.

Special features of the test structure used to simulate equipment consist of the two parallel rectangular beams separated by spacer blocks shown at position B in Fig. 1 and an adjustable mass attached to the upper beam center. The lower beam was attached to a supporting fixture at $D$, the equipment base or foundation, while the supporting fixture was bolted to four mounting points on a shock machine support fixture. Two different stiffness parameters for this test structure could be chosen by having the spacer blocks in either position A or position B between the beams. Stiffness could be varied while keeping the mass constant and vice versa. Base motions were measured by the velocity sensor directly under the lower cross beam from which shock spectra were computed.

Variation of test structure stiffness resulted in the shock spectra shown in Fig. 2. The stiffer configuration $\mathrm{A}$ (higher fixed-base frequency, $f_{1 \mathrm{~A}}$ ) gave a different peak in the shock spectrum than the less stiff configuration B (lower fixed-base frequency, $f_{1 \mathrm{~B}}$ ) because the natural frequencies 

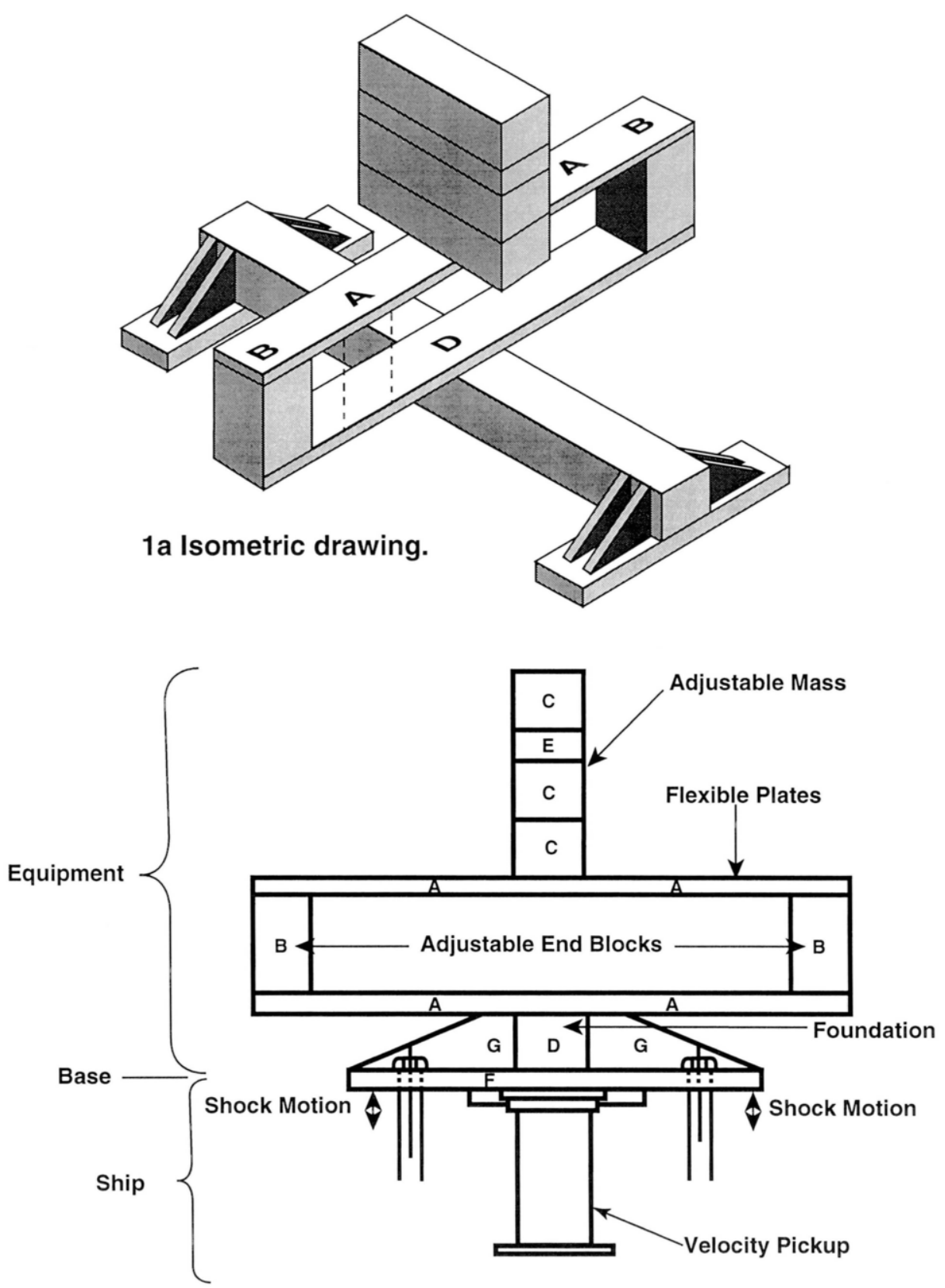

1b Arrangement of experiment

FIGURE 1 Structure used for equipment-ship interaction experiment shown in (a) an isometric drawing with (b) experimental arrangement. Adapted from O'Hara (1961).

of the entire dynamical system of the machine and experimental structure changed. The test structure frequencies (equipment fixed-base natural frequencies) at which one would normally read shock levels from the spectrum for calculation of stresses are shown to be the point at the vertical dashed lines just above the frequency scale of Fig. 2. They are not near the spectrum peaks at all.
This illustrates the potential for overdesign from using peaks or envelope shock spectra. Suppose the shock spectrum from configuration $\mathrm{B}$ is the spectrum that is used to design equipment with the same mass but different stiffness as that of configuration $\mathrm{A}$. One goes to frequency $f_{1 \mathrm{~A}}$ (fixed-base frequency of the first normal mode of configuration A) and the spectrum level read would be on the peak of the configuration $\mathrm{B}$ shock 


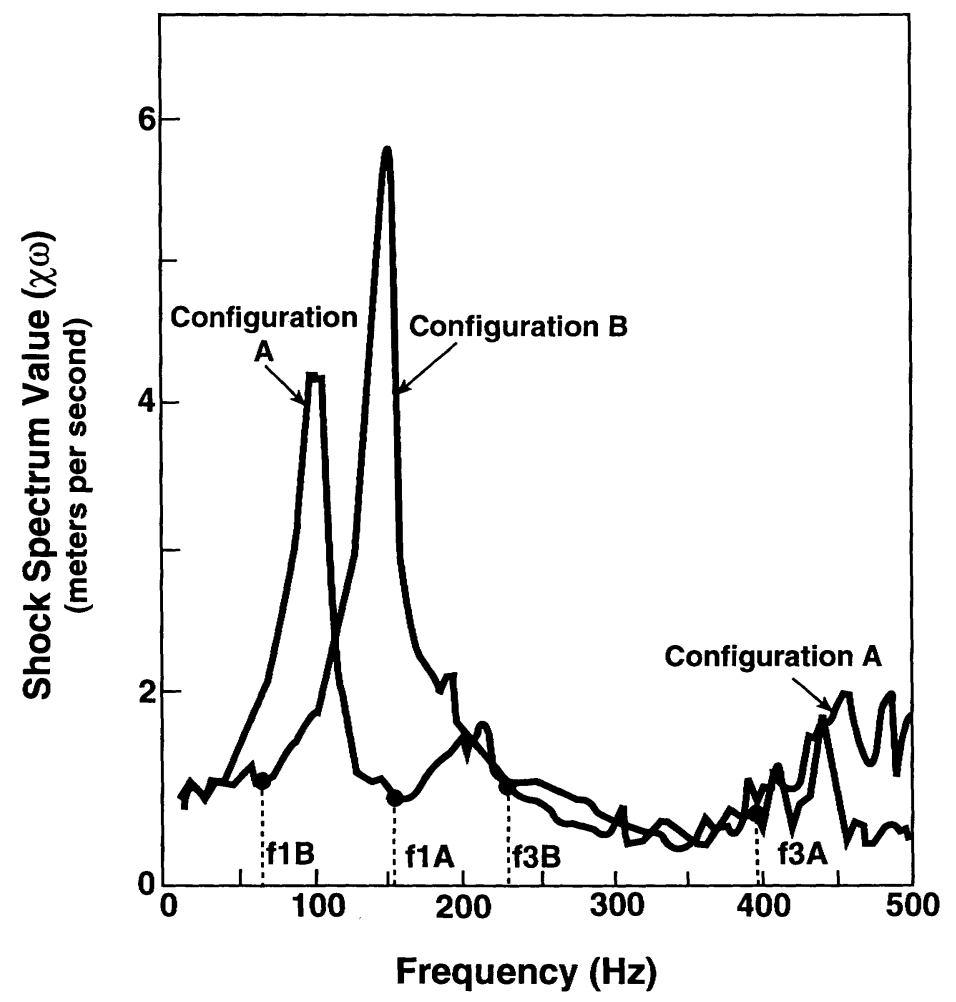

FIGURE 2 Effect of varying equipment stiffness on shock spectra. Adapted from O'Hara (1961).

spectrum. Because the correct design value at $f_{\mathrm{IA}}$ is on the configuration A shock spectrum, overdesign by a factor of more than 9 would result.

The composite spectra for the six tests in configuration A are shown in Fig. 3. The black dots are shock values at the equipment fixed-base (normal mode) natural frequencies. The shifting of the dominant peaks for very small incremental changes $(6.3 \mathrm{~kg} / 17 \mathrm{lb})$ in load can be seen.

The maximum and minimum envelopes from all variations of mass and stiffness in this experiment are shown in Fig. 4. The black dots on the figure are the shock spectrum values at all of the fixed-base natural frequencies that are the proper ones to use in calculating maximum stress in the equipment. These correct values lie much closer to the minimum envelope than to the envelope of the peaks, which was a basic criteria for design prior to this experiment. White (1966) reported this effect to the Tokyo Earthquake Conference of 1966.

An easy way to understand this is to look at a small dynamical chain in Fig. 5. This example shows how to characterize the dynamic response of part of the dynamical chain by using the "base motion."
Suppose Fig. 5(a) is the actual equipmentvehicle structure responding to an excitation $F(t)$ and the response $Y_{1}$ is desired. In each subsystem of Fig. 5 the masses and springs are identical to those of Fig. 5(a). It is also assumed that the base motion of each subsystem is identical to the corresponding motions in Fig. 5(a). $Y_{1}$ can be found from Fig. 5(a) by using $F(t)$ as the input and the system frequencies $0, \alpha_{1}, \alpha_{2}$, and $\alpha_{3}$. It can also be found from Fig. 5(b) from the known base motion $Y_{4}$ and the fixed-base normal mode frequencies $\beta_{1}, \beta_{2}$, and $\beta_{3}$. It can also be found from Fig. 5(c) with the known or measured base motion $Y_{3}$ and the fixed-base frequencies $\gamma_{1}$ and $\gamma_{2}$. Then $Y_{1}$ can also be found from the single degree of freedom system in Fig. 5(d) using only $Y_{2}$ and its frequency $\omega$. The frequencies used in the calculations, 0 and $\alpha, \beta, \gamma$, and $\omega$ are in general all different, but the response $Y_{1}$ must be the same in all cases. A simple mathematical derivation proving this point can be found in appendix $\mathrm{A}$ of O'Hara and Petak (1968).

Now suppose a time history record was obtained at each base motion $Y$ in Fig. 5 and the Duhamel integral solution was obtained for a dense set of frequency responses at each base 


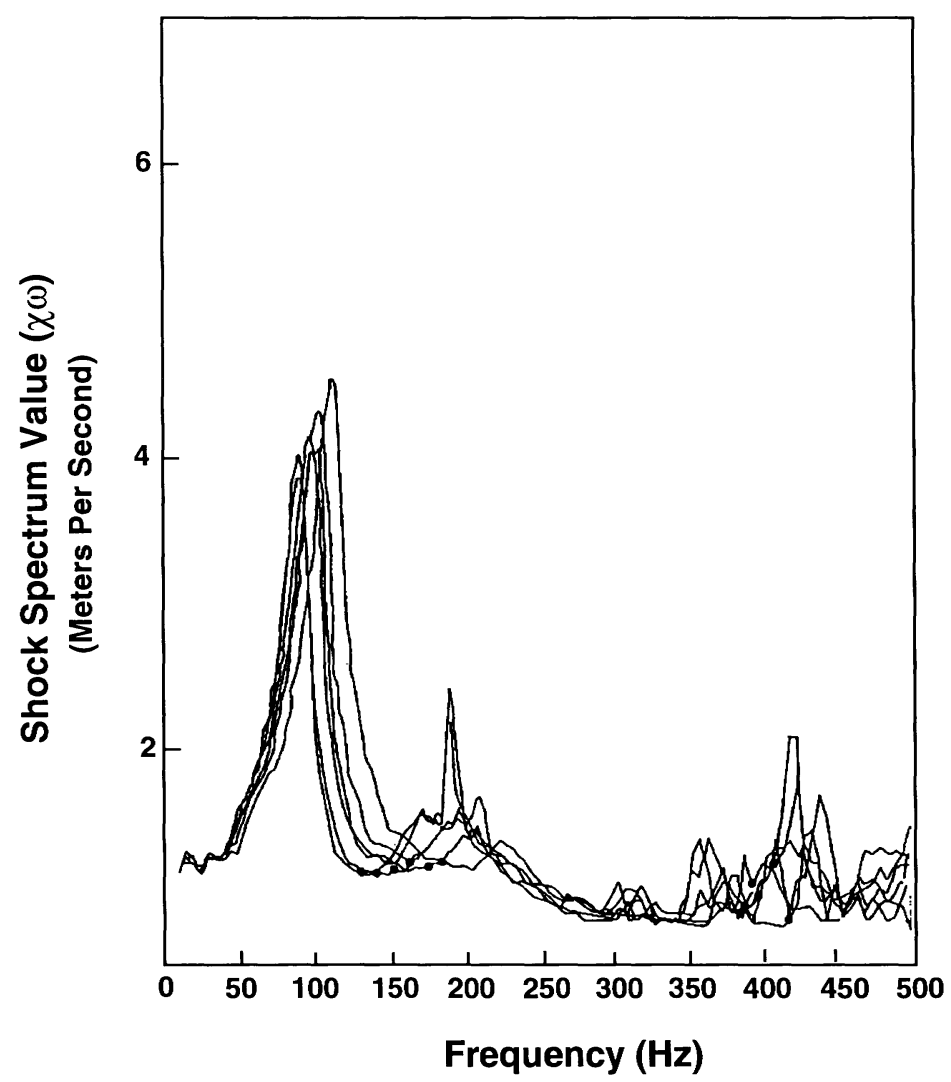

FIGURE 3 Composite of all shock spectra measured for configuration A. Adapted from O'Hara (1961).

to form a shock spectra. In general for each $Y$, something like Fig. 6 would be the result. There would be three predominate peaks at frequencies $\alpha_{1}, \alpha_{2}$, and $\alpha_{3}$ that are the actual system natural frequencies, are real, and not at the fixed-base frequencies that are only used in calculations. Because the spectrum values useful for calculating the response $Y_{1}$ in all cases do not coincide with the peaks and hence do not have the obvious resonance build-up effect, they must lie elsewhere. Thus, any upper enveloping of sets of shock spectra from different real systems will be controlled by the system frequencies and not the fixed-base frequencies used in calculations. That is, the peaks define the upper envelope and fiducial limit curves and they overwhelm the valleys where the real design values lie.

The mechanism by which equipment response determines shock input levels is somewhat similar to that of the elementary vibration absorber that suppresses the motion at its attachment point at its fixed-base natural frequency. This is also true for vibration absorbers with more than 1 degree of freedom.

\section{Modal Effective Mass and Shock Design Values}

Around 1957 the set of field trials data was then reexamined using only those points corresponding to the fixed-base frequencies of the equipment in place during the actual shock motion. The candidate values useful for a shock spectrum had dropped considerably. The situation was still murky (Belsheim and Blake, 1957) because of scatter in the data.

It was then realized that the so-called after or residual spectrum is really the magnitude of the Fourier transform of the base shock motion (O'Hara, 1959b) and would tend to be a minimum at the required frequencies. This discovery and the definition of modal effective mass permitted further progress.

If the Fourier transform integral is written as

$$
H(t)=\frac{2}{\Pi} \int_{0}^{\infty} \int_{0}^{T_{0}} H(\tau) \cos \omega[t-\tau] d \tau d \omega
$$




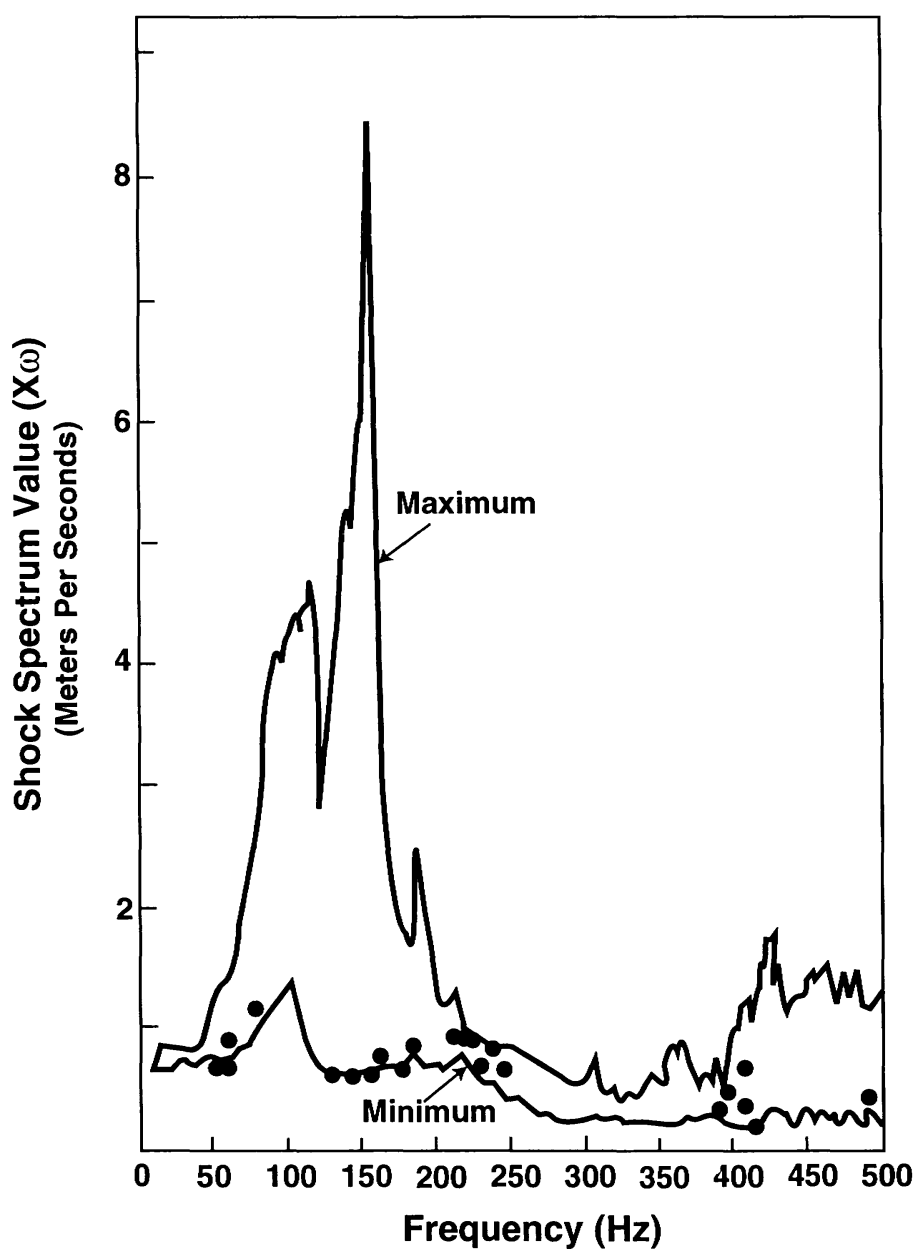

FIGURE 4 Comparison of shock values for design, shown by the dots, to the maximum envelope and minimum envelope of all shock spectra measured for configurations $A$ and B. Adapted from O'Hara (1961).
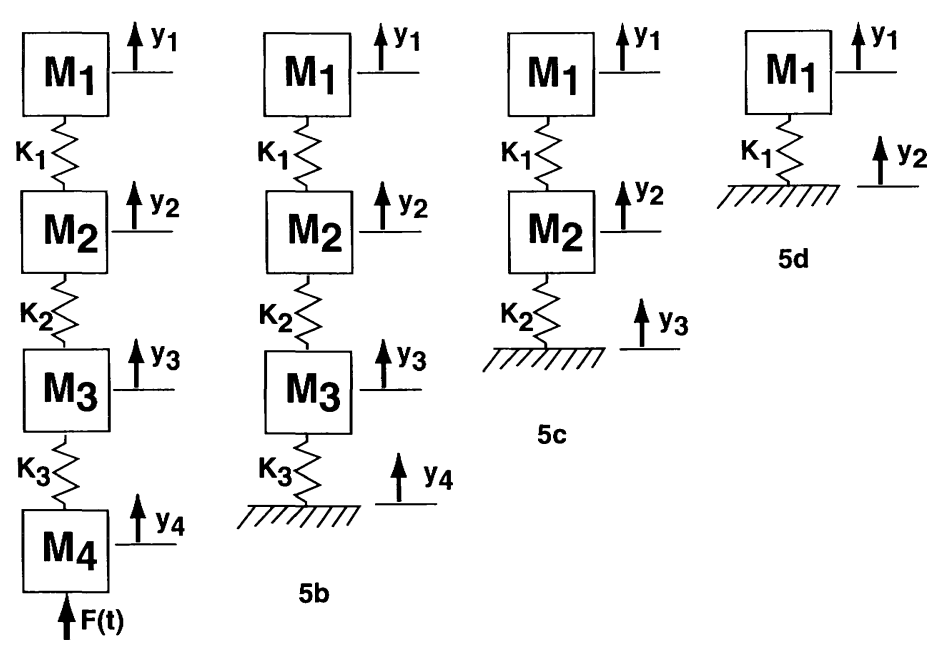

5a

FIGURE 5 (a) Dynamical chain system contains (b-d) three possible subsystems. Each has different fixed-base natural frequencies, which in turn differ from the system frequencies of (a). 


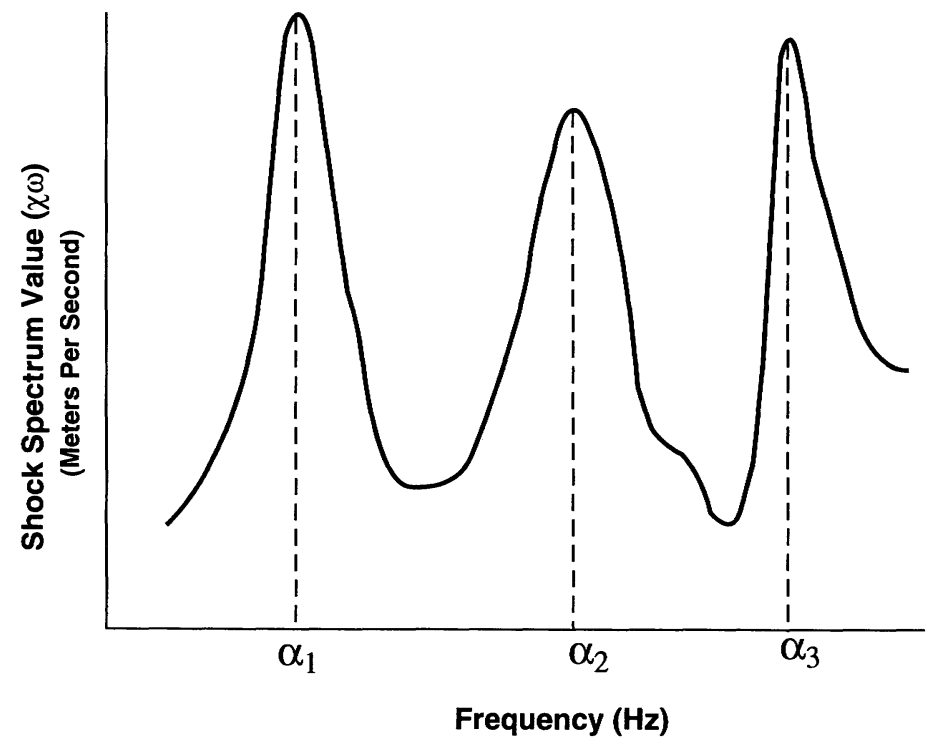

FIGURE 6 Response at system natural frequencies tends to form the peaks in a shock spectrum, while response at subsystem fixed-base natural frequencies tend to form the valleys in a shock spectrum.

then the inner time integral is also a Duhamel integral evaluated at $T_{0}$, considered to be the end of the record. This is commonly called the residual spectrum if its magnitude is plotted as a function of frequency. It is important to note that because the fixed-base frequency components are absent in the record, they will not appear in the transform but will have a value very nearly zero in the measured data.

As for the modal effective mass, it is perhaps most easily explained by reference to Figs. 7 and 8. Imagine the ship and equipment system to be

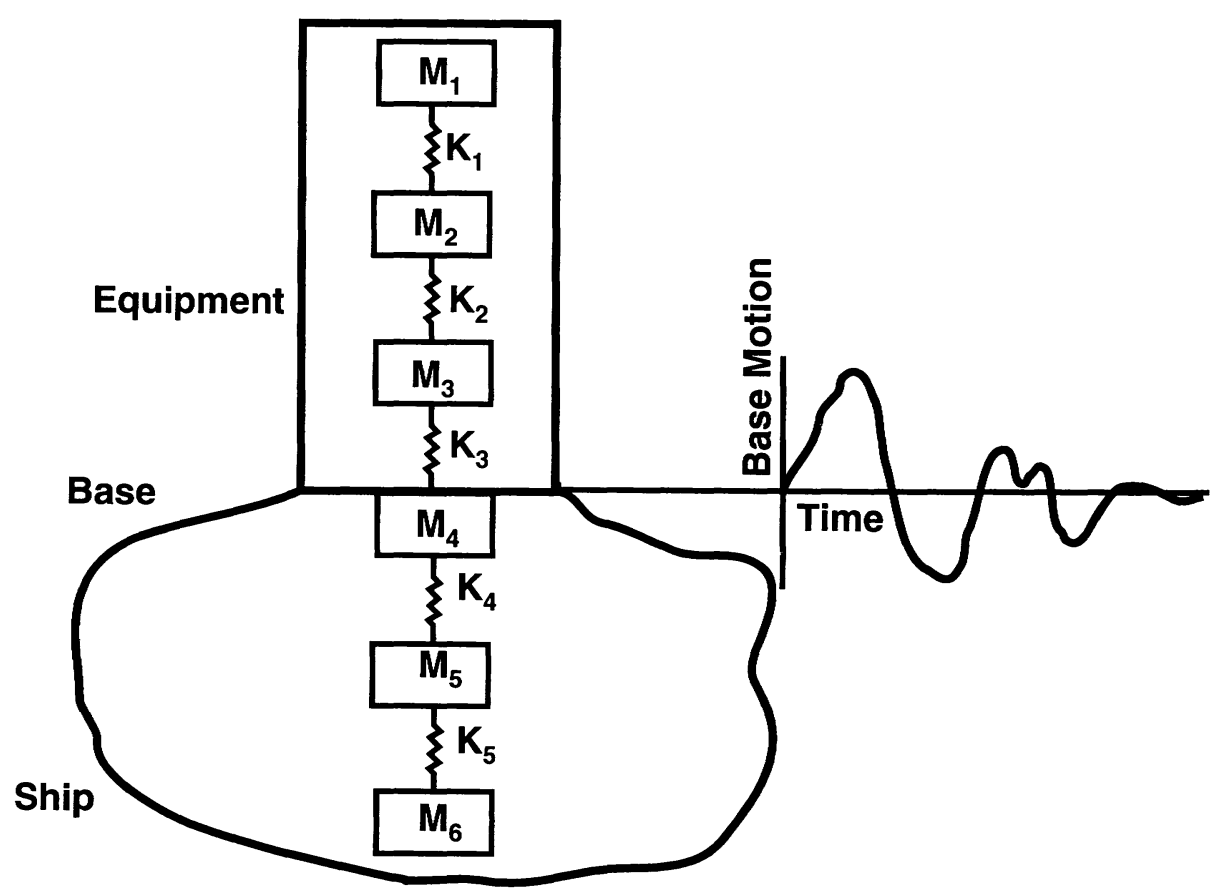

FIGURE 7 Dynamical chain representation of the equipment-ship subsystem. Adapted from Remmers (1983). 


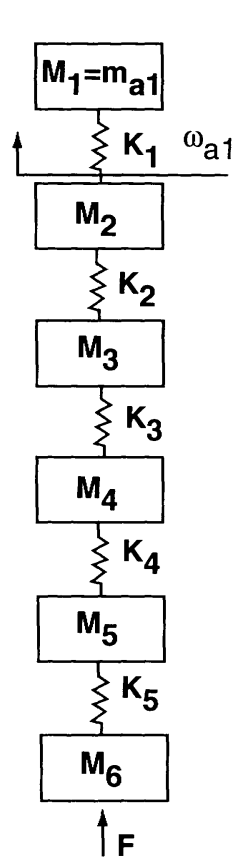

$8 a$

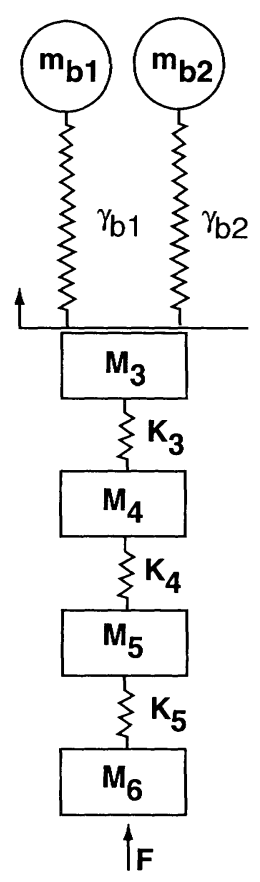

8b

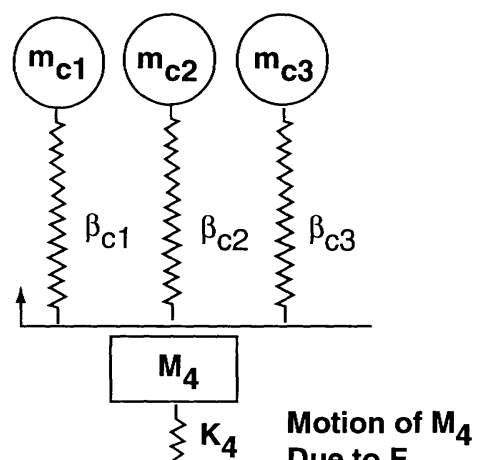

Due to $F$

Identical for (a), (b), and (c)

FIGURE 8 Illustration of replacing parts of a structure with normal mode models as used to investigate interaction effects.

represented by a dynamical chain as shown in Fig. 7, where an explosion in the water external to the ship excites the system and the response motion at the base of the equipment is recorded as shown. This system, for purposes of investigating interaction effects, can be represented by the three dynamical chains of Fig. 8, each of which is different. If the fixed-base frequencies and modal effective mass values of $m_{b 1}$ and $m_{b 2}$ are chosen properly, the response of system $a$ and $b$ will be identical for masses 3, 4, 5, and 6. Figure 8c moves one step further on the dynamical chain where again the motions of masses 4,5 , and 6 will be the same in all three systems if the masses $m_{c 1}, m_{c 2}$, and $m_{c 3}$ are properly chosen along with the corresponding fixed-base frequencies. The equation for modal mass is

$$
m_{a}=\frac{\left[\sum_{i} M_{i} \bar{X}_{i a}\right]^{2}}{\sum_{j} M_{j} \bar{X}_{j a}^{2}},
$$

where the $\bar{X}_{i a}$ is a normal mode shape value at $i$ in mode $a$. (Note that this is not the "generalized mass" that is found in eigenvector analysis.) An- other way to view Eq. (2) is that it demands that the inertial forces across the equipment-vehicle boundary be the same in all cases, for any arbitrary motion.

This is interesting in that the inertia present in each fixed-base normal mode can be used in an analysis and is a measure of the resistance to motion. This is physically intuitive in that it is easier to accelerate a light structure than a heavy one, and this showed up in the data. The sum of all the modal masses must equal the equipment mass, and a partial sum showed its usefulness in deciding on the number of modes to use.

A procedure for generating shock design values (Cunniff and O'Hara, 1989) using modal effective mass is now described. If two graphs are made for a set of the fixed-base frequency response data points, one for absolute acceleration and one for pseudovelocity (relative displacement scaled by radian frequency), each graph as a function of modal effective mass, then the upper envelope limit lines will slope downward with increasing modal mass. The graphs might appear as in Fig. 9. Now if the shock design value for a mode whose modal effective mass is $m_{a}$ and modal frequency, $f_{a}$, is desired, each graph will provide a value. The maximum absolute acceleration $A$ is 
9a Max Absolute Acceleration Data

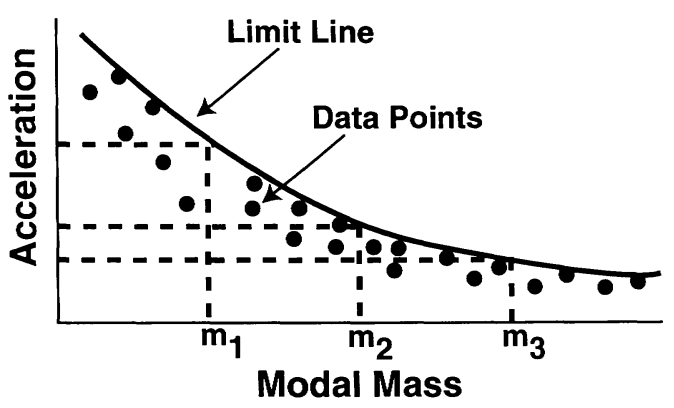

\section{9c Shock Design Levels}

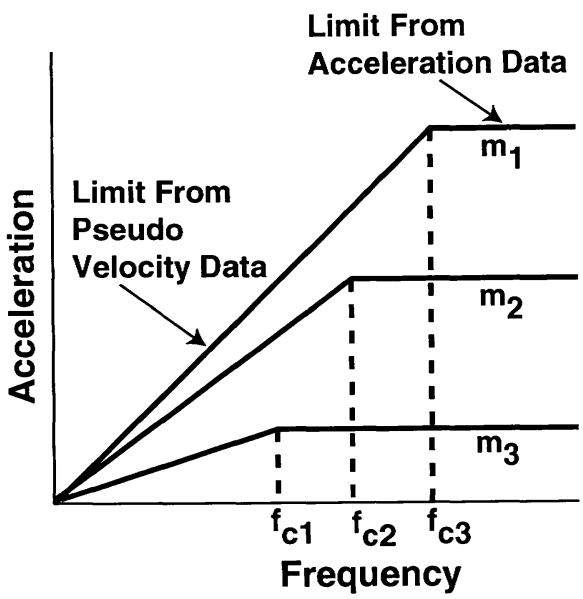

9b Max Pseudo Velocity Data

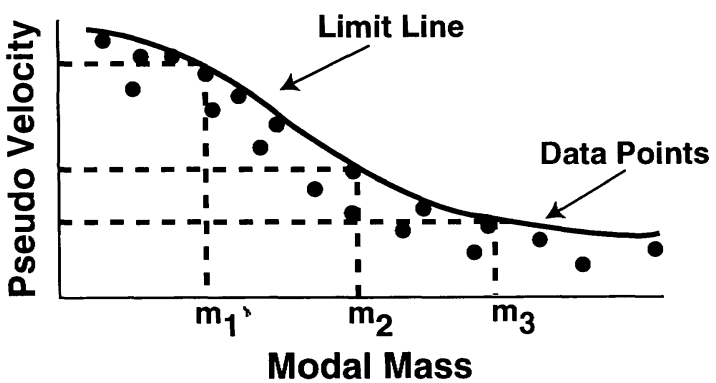

Frequency

FIGURE 9 Combining the (a) maximum shock response absolute acceleration shown with the (b) maximum shock response pseudovelocity, $(\mathrm{X} \omega)$, forms (c) a design input curve for specific modal masses $m_{1}, m_{2}, m_{3}$, etc.

related to the spectral pseudovelocity $V$ by the simple relation $A=2 \pi f_{a} V$, where $f_{a}$ is a fixedbase frequency. Thus, we have two sources of design limiting acceleration obtained from the same set of measured data. The lessor of the two values is chosen because the sloping line for frequencies greater than the cut-off frequency, $f_{\mathrm{c}}$, is greater than any limiting acceleration found from Fig. 9(a). Also the horizontal line in the frequency range below $f_{\mathrm{c}}$ is a value greater than any acceleration obtained from Fig. 9(b). Thus, a set of curves for several modal masses (weights) can be constructed as illustrated in Fig. 9(c). In practice only two numbers need to be known for each modal mass.

This section of the article sketched how the DDAM method evolved from difficulties with envelope spectra, fiducial limit spectra, and averaged spectra to a spectral technique that uses the fixed-base natural frequencies and modal effective masses as design parameters to account for the equipment structural interaction. The use of "time history" of the input motion transient for design, in place of the input spectra, was also examined during this era.

\section{Transient Design Analysis Approach}

The use of "typical time history" motions and "specific time history motions" were studied and are reviewed here. The problem was, as expected, the same as for shock spectra where it was necessary to account for equipment-vehicle interaction effects. Returning to Fig. 2 reveals that if the motion time history for configuration $B$ was used for the analysis of an equipment represented by configuration $\mathrm{A}$, then an input 9 times too great would be used for the first mode. The reason is the inherent assumption that the motion of the base (attachment point) is unaffected by whatever structure happens to be there, thus ignoring the interaction effects. The same limitation would happen if a machine were constructed to enforce a motion on a real or a model structure. Thus, using the motion of configuration B to design by the process of design, build, test, redesign, test, etc. would not result in an acceptable structure like configuration $\mathrm{A}$, but rather a structure very much like configuration $B$.

The Fourier transform is also the link between shock motions and steady-state vibrations be- 
cause the steady-state vibration spectrum including phase is the transform of the response to impulse. Norton's form of Thevinen's theorem, normally associated with steady state, can be used to produce a new transform of base motion, showing the effect of adding or replacing a structure.

The route of prescribing motions on the basis of Fourier spectrum magnitude only can produce different responses during the time of the driven portion of the massless oscillator response that produces the shock spectrum value. It was demonstrated by O'Hara (1973) that the Fourier transform magnitude of a motion that ends in finite time is not unique. That is to say, it is possible to define a function $h(t) \neq g(t)$ other than $h(t)=$ $-g(t)$ such that its Fourier transform magnitude is identical with $g(t)$. The operation $h(t)=$ $g\left(T_{0}-t\right)\left(T_{0}\right.$ is the end of the record) rotates $g(t)$ about $T$ and shifts the new origin to $T_{0}$. The two functions, while having identical residual spectra and autocorrelation functions, have different shock spectra and cross correlations.

To sum up, the several shortcomings of prescribing design inputs in terms of transient motions as described above prevented their general use in naval applications.

\section{Description of Environment}

For some naval applications severity of the shock disturbance is characterized in terms of energy flux density and/or other parameters caused by the pressure wave on the surface of the target (ship). Because local effects are important, separate input values are determined for general locations on the ship that differ in intensity (hull mounted, deck mounted, topside, etc.). Shock design values are described by vertical, athwart ship, and fore and aft directions one at a time, not the three simultaneously. It turns out not only are DDAM inputs not highly sensitive to charge size and target location for a given severity, but they also contain the effects of surface cut-off, bubble pulses, etc., because these effects were included in the original data. This is a desirable attribute for a design method.

Both DDAM and the earthquake design methods evolved from the work of Biot. The goal of each of these design methods was the same. However, the great disparity in the shock levels to be withstood required a greater degree of understanding than was available in th 1950s and re- sulted in an effort in which basic research was paramount to success.

\section{Combining Stresses, Deflections, etc.}

The modal summation technique of earthquake design usually used the statistical expected value of all the modal stresses,

$$
\begin{aligned}
p_{\mathrm{EQ}} & =\sqrt{a^{2}+b^{2}+c^{2}+\cdots} \\
& =a \sqrt{1+\frac{b^{2}+c^{2}+\cdots}{a^{2}}},
\end{aligned}
$$

which is the largest mode and a correction. This is less conservative than DDAM. With DDAM the stress, deflection, etc. is the largest modal value plus the statistical expected value of the remaining stresses at the time of occurrence of the largest modal value.

$$
\begin{aligned}
p_{\mathrm{DDAM}} & =a+\sqrt{b^{2}+c^{2}+\cdot \cdot+q^{2}+\cdots} \\
& =a+b \sqrt{\frac{1+c^{2}+d^{2}+\cdot \cdot \cdot+q^{2}+\cdots \cdot}{b^{2}}},
\end{aligned}
$$

where $a, b, c, \ldots$, are the modal stresses and $a>b>c>\cdots>q>\cdots$. This formula is, in effect, the largest mode plus the second largest mode with a correction. This generic class of formulas, when applied to normal mode analysis, is generally called the NRL sum (O'Hara and Cunniff, 1963).

\section{Survive Failure Criteria}

DDAM predicts the maximum stresses and deflections in the equipment that are then compared to engineering values for yield stresses and maximum permitted deflections that will allow the equipment function to continue.

Equipment design must be possible in a practical sense, not just in theory. Not only is it possible to set the shock values so high that the equipment cannot be designed, but it can also be set so low that any design method is ineffective.

Adjustments in failure criteria can be made in several ways. One such criteria is to use allowable stresses slightly higher than yield for bending members by means of an efficiency factor (Belsheim and O'Hara, 1960, 1961), or the standard engineering yield can be used with a reduction in shock design values. 


\section{Validation and Verification}

The shock design values used in DDAM were validated by measurements during full scale ship shock trials. The procedure was used to check the designs of equipment since 1960 and became a Navy design requirement in 1961 (Belsheim and O'Hara, 1961; O'Hara and Belsheim, 1961). The veracity of DDAM was demonstrated by shock trials and by the survival of DDAM designed equipment during wartime attack. After some initial resistance to implementation as a design procedure by the Navy, many engineers were trained to use it and it continues to be routinely used to this day.

\section{FOLLOW-UP DEVELOPMENTS}

\section{Determination of Normal Mode Parameters}

In the early 1960 s an attempt to measure the fixedbase frequencies of equipment with the then common technique failed. That technique was to excite the top mass of a dynamical chain with a vibration generator with the peaks in the ratio of the measured motion of the top mass to the measured motion of the base indicating the fixedbased natural frequencies. In an analytical study of how to measure fixed-base natural frequencies that followed, Petak and Kaplan (1964) discovered that the vibration excitation could only be applied to the base of the equipment (or in the basement of a building) or below on the vehicle in order to determine the proper normal mode fixed-base frequencies. Placing the exciter at the top mass of the dynamical chain (top of equipment of building as shown in Fig. 7) guarantees that the fixed-base natural frequencies cannot be found. To validate the analysis the experimental measurement of the fixed-base normal mode natural frequencies of an equipmentlike structure, while the equipment was part of a larger dynamical system, was made by Remmers and Mayo (1967).

O'Hara and Remmers (1969) conducted the first known successful measurement of the modal masses of an equipment test structure, thus confirming its effects.

Modal mass and frequency parameters can also be determined, to some extent, from measured structural response transients as described by O'Hara (1959b) and by Cunniff and O'Hara (1988).

\section{Effect of Damping on Shock Spectrum Values}

Damping can affect measured shock spectra. An analytical study of these damping effects on spectra from a dynamical chain by Cunniff and Collins (1968) is shown in Fig. 10. The shock spectra from the motion of mass 4 was computed using three levels of damping. The peaks that correspond to the system frequencies are strongly affected. But the spectrum values at the fixed-base natural frequencies, which are shown as dashed arrows, were not changed very much. Thus, shock design values are reduced by only a small amount by the presence of damping and can be neglected in most cases. Also, the amount of damping present in the modal oscillators can be deduced from the measured transient response as shown by O'Hara and Cunniff (1988).

\section{Effect of Nearby Equipment Structures}

The significance of the effect of nearby equipment structure or a second mode of the equipment upon shock design values was studied by O'Hara and Petak (1968). Shock design values for simple equipment by itself were compared to the shock design values for the same simple equipment with a generally comparable equipment structure nearby. This initial, somewhat crude exploratory study showed design input levels to be somewhat affected but indicated the shock design values are relatively insensitive to a second mode or nearby equipment.

\section{Effect of Yielding on Shock Design Values}

When measuring severe shock motions there should be concern over possible yielding in the structure of either the ship or the equipment, especially when using measured responses to establish shock design values. Studies of this phenomena by Mentzer and Cunniff (1968) and by O'Hara and Huang (1978) are evaluated in Fig. 11, which illustrates the results of increasing the amount of yielding in the equipment. (The term equipment by itself includes the support by which equipment is attached to the vehicle, that is, the support or connection to the vehicle is on the equipment side of the equipment-vehicle system.) The shock motion was assumed to produce $882 \mathrm{MPa}(90 \mathrm{ksi})$ stress in the connection. Equipment supports that differ only in yield stresses ranging from 294 to $1470 \mathrm{MPa}$ (30 and $150 \mathrm{ksi}$ ) were considered. 

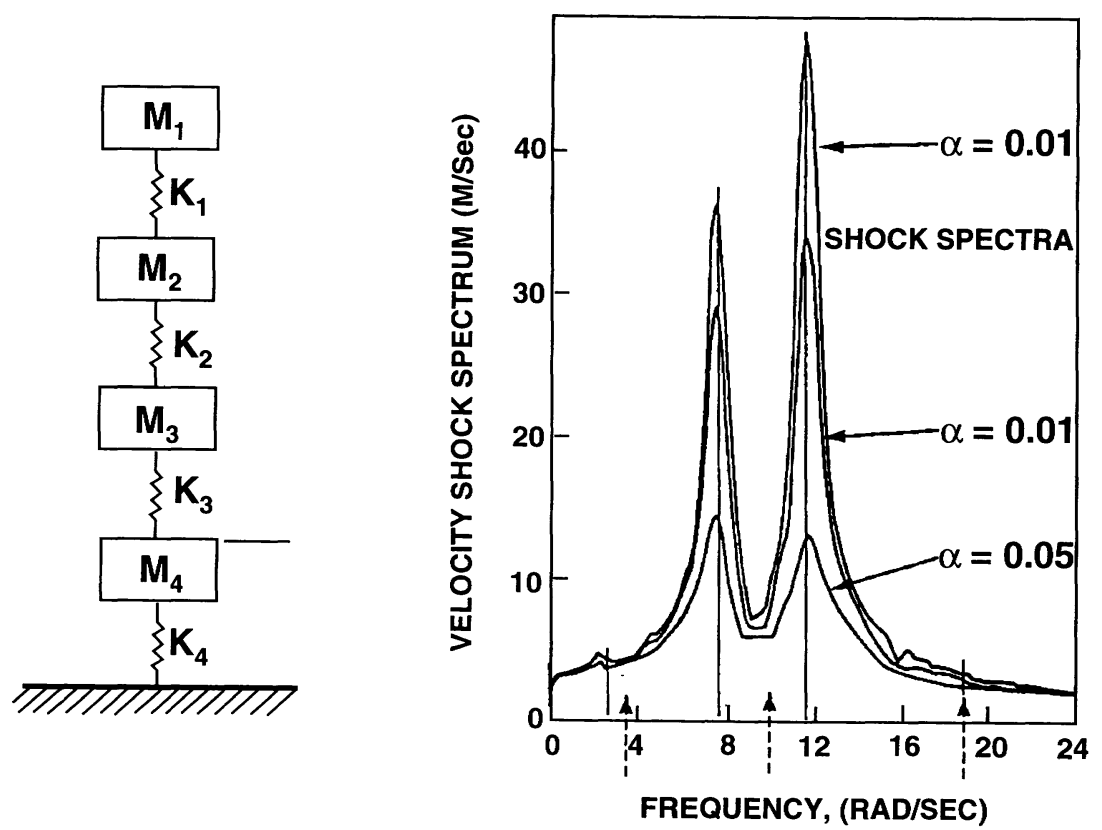

FIGURE 10 Effect of damping on shock design values. Adapted from Cunniff and Collins (1968).

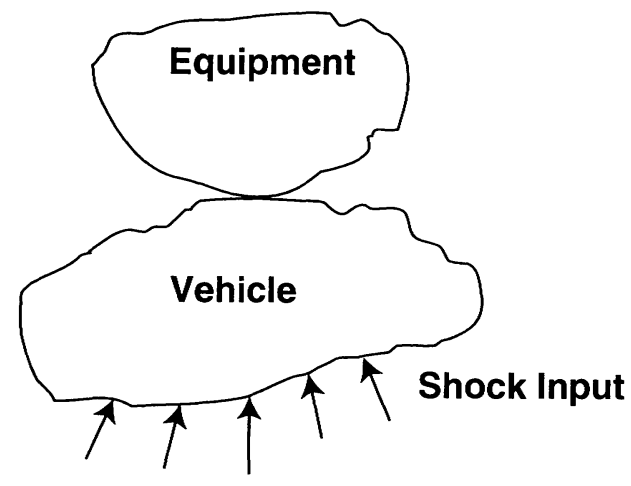

Thus the weight remained constant. When the equipment supports remained elastic (980 and $1470 \mathrm{MPa}$ cases) the elastic shock design value resulted. When yielding in the equipment support occurred, the shock design values were higher than the elastic case, with the highest value for the largest amount of yielding. If the shock design values were determined from measurements where equipment support yielding occurred, the design levels were more severe than necessary. Conversely, however, when yielding occurred in the vehicle, the measured shock design value was lower than the elastic value.

Thus, if it were desirable to permit some yielding in the equipment support structure, the use of the values for the 294, 490, and $784 \mathrm{MPa}$ tests where yielding occurred would guarantee that it would not yield, because these values were greater than the elastic response value. Of course, if yield were to occur in the ship, vehicle, or earth, then the measured "elastic" value would be too low and some yielding would occur if these values were used to design equipment where yield is not desired.

\section{Equipment on Multiple Supports}

FIGURE 11 Effect of yielding on shock design values. Adapted from Remmers (1983).

One of the assumptions of the original DDAM is that the entire base of the equipment-support 
system moves uniformly. This means that if it is mounted on two supports or more, these supports are subjected to the same base motion at the same time. Because this is not always the case, especially for large items, three aspects of this problem were examined.

First a procedure for measuring the fixed-base natural frequencies of equipment on two supports was developed. It is significantly more complex than for a single support (Petak and O'Hara, 1966). Second, O'Hara and Belsheim (1974) investigated the problem of loading the structure by shock waves traveling through a medium resulting in a time delay between dominant shock wave loadings at separated input points. Finally, a procedure for using DDAM-like inputs to design multisupport equipment was developed by Abdelhamid and Cunniff (1991).

\section{Scaling and Testing}

It is not always practical to test at full scale or to extend data to different levels of shock intensity. Practical design methods readily lend themselves to scaling design problems, such as scaling shock design inputs measured on a submarine of one diameter to be appropriate for a second diameter. This analytical approach was used by O'Hara and Cunniff (1993) for simulation of vehicle shock tests of lightweight hull mounted equipment at different scales.

\section{Normal Mode Theory}

Normal mode relationships for unidirectional and for three and six directions of motion of elemental equipment mass were developed by O'Hara and Cunniff (1963), Cunniff and O'Hara (1965), and Fourney and O'Hara (1968). These were used as the basis for several advanced structural analysis procedures.

\section{Design of Efficient Structures}

Reduction of equipment support weight centered around defining structural efficiency as the square root of the ratio of the actual shock energy absorbed by a structure to the total energy storage capacity of the structure. An elastic method was reported by O'Hara and Cunniff (1982a), and the elastic-plastic case was shown by O'Hara and Cunniff (1982b). Significant support weight reductions were projected using these methods while assuming the equipment being supported was already hardened.

\section{MYTHS AND MISCONCEPTIONS}

Despite the fact that many years have elapsed since the inception of DDAM, some widespread myths and misunderstandings about some features of the method still prevail. They are:

- a transient dynamic analysis is unique and provides a better solution than DDAM for attacks at the same shock design severity;

- the method fails to account for fixed-based normal modes with repeated frequencies;

- if a structure has two fixed-base frequencies very close to each other, the resulting beating response is so long in time to the peak response that the combinatorial rules are not realistic;

- a very small appendage attached to large equipment can cause overly severe, erroneous values in the shock input, particularly if the appendage fixed-base frequency is about equal to a fixed-base frequency of the equipment.

Each of these was reexamined by Cunniff and O'Hara (1993) and are summarized below.

The transient analysis of a simple equipmentvehicle model was developed that replicates the damaging potential prescribed by DDAM-like design inputs. The equipment discussed therein was limited to 2 degrees of freedom, unidirectional motion, and the vehicle was composed of a lumped mass-spring combination excited by an impulse. The magnitude of solutions provided by the method clearly demonstrated that there is no unique transient model available. This reference shows this with an example where three distinct vehicles experience different impulses. The ensuing motion of each vehicle was different and generated different shock spectra. However, the shock spectrum values at the equipment fixedbase frequencies were identical in each case. Thus, transient motion inputs as used by the proposed method are not unique. Furthermore, the transient technique cannot be better than the data upon which it is based, i.e., the DDAM shock input values.

An examination of the special case of repeated natural frequencies indicated that the modal effective masses, the participation factors, and the characteristic loads are zero at these repeated 
roots. Hence, these modes do not contribute to the deflections and stresses in the DDAM analysis or in any other normal mode spectral analysis method.

In the case of closely spaced equipment fixedbase frequencies, the transient model provided responses that were composed of the system natural frequencies; therefore, they did not pose any special problems, such as a resonance build-up effect, even though the equipment frequencies were closely spaced.

It was shown that the addition of a lightweight appendage with its own fixed-base frequency about equal to the main equipment fixed-base frequency, to rather heavy weight equipment can cause failure difficulties because the normal mode parameters change. However, doing this would be a poor design practice. Nevertheless, the DDAM shock design inputs, via the modal effective mass, compensate for this poor design effort by increasing shock design values, as it should. That is to say that the structural interaction effect using modal effective mass increased the shock design values. The analysis also showed that a change in the fixed-base frequency of the appendage should avoid the large deformations that would otherwise occur and are predicted by DDAM. Further work (Cunniff and O'Hara, 1994) showed that while shock spectra are unique, systems can be devised that have nearly identical shock design values, even though their shock spectra are different.

Some authors and engineers have called the pseudovelocity form of the scaled relative displacement spectra an equivalent step change of velocity of the base. Such a claim results in a sinusoidal response after the sudden base motion. To extend this definition to say that a single step change of velocity of the base can result in two different values of the product $X \omega$ for different modes is incorrect and need not be explored further.

\section{CONCLUSIONS}

It is noteworthy that DDAM continues to be used 36 years after it was introduced. There are several primary reasons for this. DDAM is based on a thorough foundation of research and was developed to be easy to use, accurate, and fast so that it can be readily used by ship designers. The method can be successfully exercised within the time constraints of ship acquisition schedules.
The method was successfully validated in several ways including DDAM designed equipment that survived actual attacks.

Because the background basic research was already done and was well documented, application in other structural dynamics situations, such as rockets, earthquake, vibration testing, etc., should be explored.

\section{REFERENCES}

Abdelhamid, M. K., and Cunniff, P. F., 1991, "Shock Design Rules for Multi-Foundation Problem," Proceedings of the Thirteenth Biennial ASME Conference, Miami, FL.

Belsheim, R. O., and Blake, R. E., 1957, Effect of Equipment Dynamic Reaction on Shock Motions of Foundations, Naval Research Laboratory Report 5009, Washington, DC.

Belsheim, R. O., and O'Hara, G. J., 1960, Shock Design of Shipboard Equipment-Dynamic Design Analysis Method, Naval Research Laboratory Report 5545, Washington, DC.

Belsheim, R. O., and O'Hara, G. J., 1961, Shock Design of Shipboard Equipment-Dynarnic Design Analysis Method, Department of Navy NavShips Publication 250-423-30, Part I, Washington, DC.

Cunniff, P. F., and Collins, R. P., 1968, "Structural Interaction Effects on Shock Spectra," Journal of the Acoustical Society of America, Vol. 43, p. 239.

Cunniff, P. F., and O'Hara, G. J., 1965, Normal Mode Theory for Three Dimensional Motion, Naval Research Laboratory Report 6170, Washington, DC.

Cunniff, P. F., and O'Hara, G. J., 1988, "Modal Characteristics from Structural Transient Response Data,' International Journal of Analytical and Experimental Modal Analysis, Vol. 2, pp. 180-191.

Cunniff, P. F., and O'Hara, G. J., 1989, “A Procedure for Generating Shock Design Values," Journal of Sound and Vibration, Vol. 134, pp. 155-164.

Cunniff, P. F., and O'Hara, G. J., 1993, "Some Thoughts on Perceived DDAM Problems,' Proceedings of the Eleventh International Modal Analysis Conference, pp. 1447-1453.

Cunniff, P. F., and O'Hara, G. J., 1994, "Feasibility of a Transient Dynamic Design Analysis Method," Shock and Vibration, Vol. 1, pp. 241-251.

Fourney, W. L., and O'Hara, G. J., 1968, "Normal Modes and Natural Frequencies of Combined Structures,' Journal of the Acoustical Society of America, Vol. 44, pp. 1220-1224.

Mentzer, W. R., Jr., and Cunniff, P. F., 1968, Yielding Effects on Shock Spectra, Shock and Vibration Bulletin 36, Part II, The Shock and Vibration Information Center, Naval Research Laboratory, Washington, DC. 
O'Hara, G. J., 1958, Effect upon Shock Spectra of the Dynamic Reaction of Structures, Naval Research Laboratory Report 5236, Washington, DC.

O'Hara, G. J., 1959a, Shock Spectra and Design Shock Spectra, Naval Research Laboratory Report 5386, Washington, DC.

O'Hara, G. J., 1959b, “Impedance and Shock Spectra," Journal of the Acoustical Society of America, Vol. 31, p. 1300.

O'Hara, G. J., 1961, “Effect upon Shock Spectra of the Dynamic Reaction of Structures," Journal of the Society for Experimental Stress Analysis, Vol. 1, No. 5, pp. 145-151.

O'Hara, G. J., 1967, “Mechanical Impedance and Mobility Concepts," Journal of the Acoustical Society of America, Vol. 41, pp. 1180-1184.

O'Hara, G. J., 1973, A Note on the Non-Uniqueness of Fourier Transform Magnitudes, Reports of Naval Research Laboratory Progress, February, Washington, DC.

O'Hara, G. J., and Belsheim, R. O., 1961, Shock Design of Shipboard Equipment Part II-Interim Shock Design Inputs for Submarine and Surface Ship Equipment, Department of Navy NavShips Publication 250-423-31, Washington, DC.

O'Hara, G. J., and Belsheim, R. O., 1963, Interim Design Values for Shock Design of Shipboard Equipment, Naval Research Laboratory Memo Report 1396, Washington, DC.

O'Hara, G. J., and Belsheim, R. O., 1974, Treatment of Multi Foundation Data to Obtain Shock Spectra for Design Information, Reports of Naval Research Laboratory Progress, August, Washington, DC.

O'Hara, G. J., and Cunniff, P. F., 1963, Elements of Normal Mode Theory, Naval Research Laboratory Report 6002, Washington, DC.

O'Hara, G. J., and Cunniff, P. F., 1982a, Efficient Elastic Design of Small Foundations, Naval Research Laboratory Memorandum Report 4886, Washington, DC.

O'Hara, G. J., and Cunniff, P. F., 1982b, Efficient Elastic-Plastic Design of Small Foundations, Naval Research Laboratory Memorandum Report 4918, Washington, DC

O'Hara, G. J., and Cunniff, P. F., 1985, “The Shock
Spectrum Dip Effect,' Journal of Sound and Vibration, Vol. 103, pp. 311-321.

O'Hara, G. J., and Cunniff, P. F., 1988, "An Equivalence of Response for the Mechanical Oscillator," Journal of the Acoustical Society of America, Vol. 89, No. 4, pp. 180-191.

O'Hara, G. J., and Cunniff, P. F., 1993, "Scaling for Shock Response of Equipment in Different Submarines," Shock and Vibration, Vol. 1, pp. 161-170.

O'Hara, G. J., and Huang, H., 1978, Yielding Effects on Design Shock Spectra, Naval Research Laboratory Memorandum Report 3862, Washington, DC.

O'Hara, G. J., and Petak, L. P., 1968, Effect of a Second Mode and Nearby Structures on Shock Design Values, Naval Research Laboratory Report 6676, Washington, DC.

O'Hara, G. J., and Remmers, G. M., 1969, Measurement of a Structure's Modal Effective Mass, Shock and Vibration Bulletin 39, Naval Research Laboratory, Washington, DC.

O'Hara, G. J., and Sweet, A. L., 1960, Methods for Design of Structures, Reports of Naval Research Laboratory Progress, July, Washington, DC.

Petak, L. P., and Kaplan, R. E., 1964, Resonance Testing in the Determination of Fixed-Base Natural Frequencies of Shipboard Equipment, Naval Research Laboratory Report 6176, Washington, DC.

Petak, L. P., and O'Hara, G. J., 1966, Determination of Fixed-Base Natural Frequencies of Dual Foundation Shipboard Equipments by Shake Tests, Naval Research Laboratory Report 6451, Washington, DC.

Remmers, G. M., 1983, Evolution of Spectral Techniques in Navy Shock Design, Shock and Vibration Bulletin 53, Part I, The Shock and Vibration Information Center, Naval Research Laboratory, Washington, DC, pp. 59-70.

Remmers, G. M., and Mayo, H. C., 1967, Experimental Technique for Determining Fixed-Base Natural Frequencies of Structures on Non-Rigid Attachment Points, Naval Research Laboratory Memorandum Report 1800, Washington, DC.

White, M. P., 1966, "The Meaning of Spectra of Earthquake Records Obtained in or Near Structures," Proceedings of the Tokyo Earthquake Conference, Summer. 

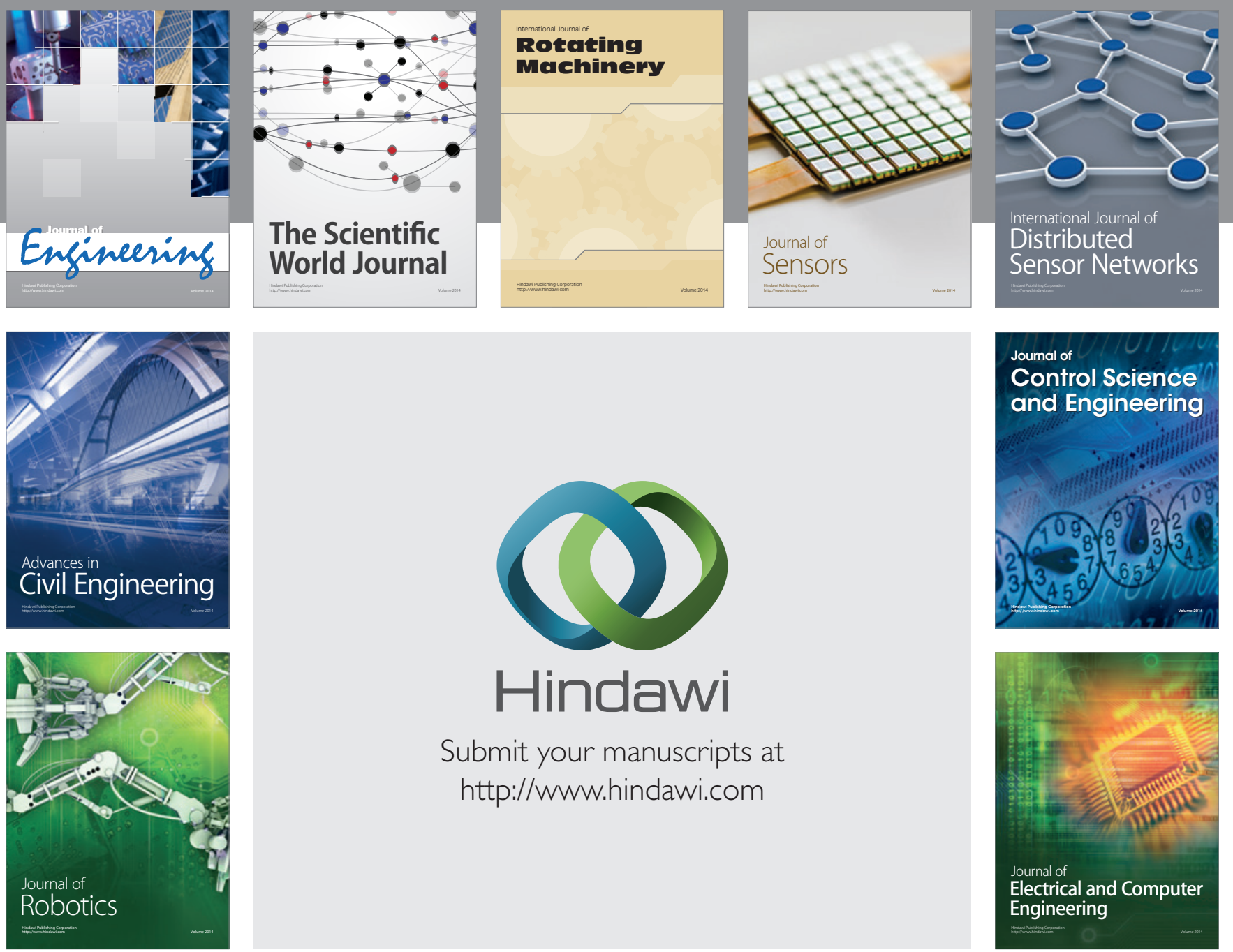

Submit your manuscripts at

http://www.hindawi.com
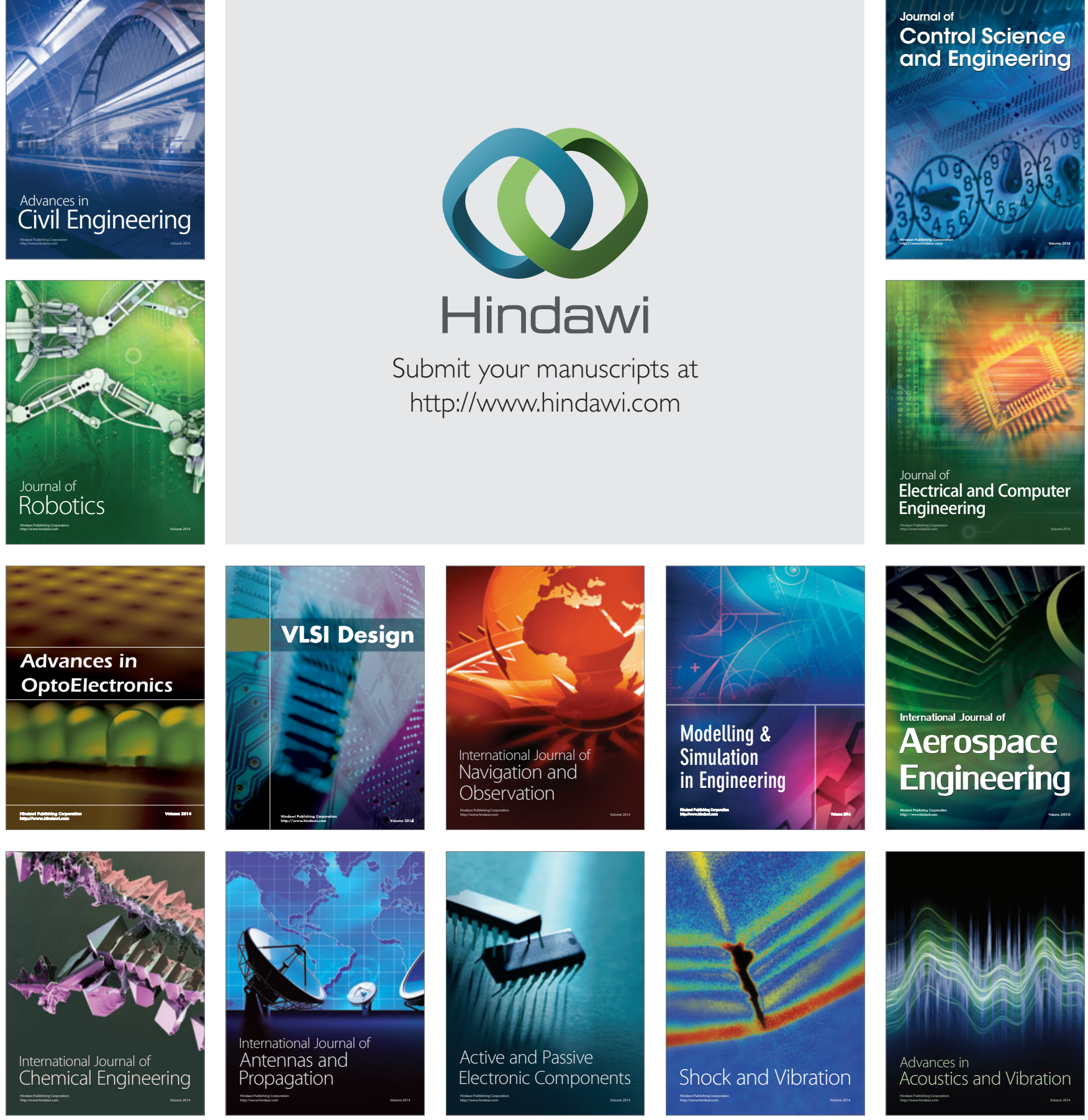